\title{
THE DECOMPOSITION GROUPS OF PLANE CONICS AND PLANE RATIONAL CUBICS
}

\author{
TOM DUCAT, ISAC HEDÉN, AND SUSANNA ZIMMERMANN
}

\begin{abstract}
The decomposition group of an irreducible plane curve $X \subset \mathbb{P}^{2}$ is the subgroup $\operatorname{Dec}(X) \subset \operatorname{Bir}\left(\mathbb{P}^{2}\right)$ of birational maps which restrict to a birational map of $X$. We show that $\operatorname{Dec}(X)$ is generated by its elements of degree $\leq 2$ when $X$ is either a conic or rational cubic curve.
\end{abstract}

\section{INTRODUCTION}

1.1. Preliminaries. We work over an algebraically closed field $\mathrm{k}$ of any characteristic. By elementary quadratic transformation we will mean a birational map $\varphi \in \operatorname{Bir}\left(\mathbb{P}^{2}\right)$ of degree 2 with only proper base points.

Definition 1.1. For an irreducible curve $X \subset \mathbb{P}^{2}$, the decomposition group $\operatorname{Dec}(X)$ of $X$ is the subgroup of $\operatorname{Bir}\left(\mathbb{P}^{2}\right)$ of all birational maps $\varphi \in \operatorname{Bir}\left(\mathbb{P}^{2}\right)$ which restrict to a birational map $\left.\varphi\right|_{X}: X \rightarrow X$.

Similarly, the inertia group $\operatorname{Ine}(X)$ of $X$ is the subgroup of $\operatorname{Bir}\left(\mathbb{P}^{2}\right)$ of all birational maps $\varphi \in \operatorname{Bir}\left(\mathbb{P}^{2}\right)$ which restrict to the identity map $\left.\varphi\right|_{X}=\operatorname{id}_{X}$.

Elements of $\operatorname{Dec}(X)$ are said to preserve the curve $X$, whilst elements of $\operatorname{Ine}(X)$ are said to fix $X$. We will write $\operatorname{Aut}\left(\mathbb{P}^{2}, X\right)=\operatorname{Dec}(X) \cap \mathrm{PGL}_{3}$ for the subgroup of linear maps $\operatorname{Aut}\left(\mathbb{P}^{2}\right)=\mathrm{PGL}_{3}$ which preserve $X$.

The focus of this paper is on the group $\operatorname{Dec}(X)$ in the case that $X \subset \mathbb{P}^{2}$ is a plane rational curve of degree $\leq 3$. In this case $X$ is either a line, a smooth conic, a nodal cubic or a cuspidal cubic.

Remark 1.2. A line $X \subset \mathbb{P}^{2}$ (resp. conic, nodal cubic, cuspidal cubic) is projectively equivalent to any other line $X^{\prime} \subset \mathbb{P}^{2}$ (resp. conic, nodal cubic, cuspidal cubic), i.e. there is an automorphism $\lambda \in \mathrm{PGL}_{3}$ with $\lambda(X)=X^{\prime}$. For rational curves of degree $\geq 4$ this is no longer true in general.

1.2. Motivation. The decomposition and inertia groups of plane curves have appeared in a number of places.

1.2.1. Decomposition and inertia groups of plane curves of genus $\geq 1$. The inertia groups of plane curves of geometric genus $\geq 2$ were studied by Castelnuovo [6], and his results were made more precise by Blanc-Pan-Vust [3]. In both articles adjoint linear systems are used to study properties of the group - a technique which does not work

2010 Mathematics Subject Classification. 14E07.

The first and second named authors are International Research Fellows of the Japanese Society for the Promotion of Sciences, and this work was supported by Grant-in-Aid for JSPS Fellows Number 15F15771 and 15F15751 respectively. The last named author gratefully acknowledges support by the Swiss National Science Foundation grant P2BSP2_168743. 
for curves of genus $\leq 1$. The inertia groups of smooth cubic curves have been studied by Blanc [2].

Decomposition groups were introduced by Gizatullin [9], who used them as a tool to give sufficient conditions for $\operatorname{Bir}\left(\mathbb{P}^{2}\right)$ to be a simple group. This group is not simple, as shown later by Cantat-Lamy [5] for algebraically closed fields, and by Lonjou [11] for arbitrary fields. The decomposition groups of plane curve of genus $\geq 2$ and some plane curves of genus 1 (smooth cubic curves and Halphen curves) are described in [4], as well as the decomposition group of rational plane curves $X \subset \mathbb{P}^{2}$ of Kodaira dimension $\kappa\left(\mathbb{P}^{2}, X\right)=0$ or 1 .

For curves $X \subset \mathbb{P}^{2}$ with $\kappa\left(\mathbb{P}^{2}, X\right)=-\infty$, the pair $\left(\mathbb{P}^{2}, X\right)$ is birationally equivalent to $\left(\mathbb{P}^{2}, L\right)$ where $L \subset \mathbb{P}^{2}$ is a line, and a description of $\operatorname{Dec}(L)$ is given by Theorem 1 below. As $X \subset \mathbb{P}^{2}$ is the image of $L$ under a birational transformation $\varphi$ of $\mathbb{P}^{2}$, we have an isomorphism $\operatorname{Dec}(X) \simeq \operatorname{Dec}(L)$, given by $\psi \mapsto \varphi^{-1} \psi \varphi$. Although it is not degree-preserving, this isomorphism shows that $\operatorname{Dec}(X)$ is not finite.

1.2.2. The decomposition group of a line. The classical Noether-Castelnuovo Theorem [7] states that the Cremona group $\operatorname{Bir}\left(\mathbb{P}^{2}\right)$ has a presentation given by:

$$
\operatorname{Bir}\left(\mathbb{P}^{2}\right)=\left\langle\mathrm{PGL}_{3}, \sigma\right\rangle
$$

where $\sigma$ is any choice of elementary quadratic transformation. The second two authors [10] have shown that an analogous statement holds for the decomposition group of a line:

Theorem 1 ([10]). Let $L \subset \mathbb{P}^{2}$ be a line. Then

$$
\operatorname{Dec}(L)=\left\langle\operatorname{Aut}\left(\mathbb{P}^{2}, L\right), \sigma\right\rangle
$$

for any choice of elementary quadratic transformation $\sigma \in \operatorname{Dec}(L)$. In particular any map $\tau \in \operatorname{Dec}(L)$ can be factored into elementary quadratic transformations inside $\operatorname{Dec}(L)$.

In this article, we present a similar theorem for conic and rational cubic curves. Uehara [13, Proposition 2.11] proves that for the cuspidal cubic $X \subset \mathbb{P}^{2}$, the elements of the subset

$$
\{f \in \operatorname{Dec}(X) \mid f \text { is an automorphism near the } \operatorname{cusp}\} \subsetneq \operatorname{Dec}(X)
$$

can be decomposed into quadratic transformations preserving $X$. Theorem 3 generalises his result to all of $\operatorname{Dec}(X)$.

1.2.3. Relationship to dynamics of birational maps. Birational maps of $\mathbb{P}^{2}$ preserving a curve of degree $\leq 3$ show up naturally when studying the dynamical behaviour of birational maps of surfaces. For instance, Diller-Jackson-Sommese [8, Theorem 1.1] show that a connected curve which is preserved by an algebraically stable element of $\operatorname{Bir}\left(\mathbb{P}^{2}\right)$ with positive first dynamical degree necessarily has degree $\leq 3$.

In their studies of automorphisms of rational surfaces, Bedford-Kim $[1, \S 1]$ explore the dynamical behaviour of the family of birational transformations $f_{a, b}:(x, y) \mapsto\left(y, \frac{y+a}{x+b}\right)$, for $a, b \in \mathbb{C}$. In particular, they focus on maps of this kind preserving a curve, and show that this curve is necessarily cubic. 
1.3. Main results. We will use Theorem 1 to deduce:

Theorem 2. Let $C \subset \mathbb{P}^{2}$ be a conic. Then any map $\tau \in \operatorname{Dec}(C)$ can be factored into elementary quadratic transformations inside $\operatorname{Dec}(C)$.

Moreover, from Theorem 2 we will deduce:

Theorem 3. Let $X \subset \mathbb{P}^{2}$ be a rational cubic and suppose that the characteristic of $\mathrm{k}$ is not 2. Then any map $\tau \in \operatorname{Dec}(X)$ can be factored into elementary quadratic transformations inside $\operatorname{Dec}(X)$.

The basic strategy used to prove both Theorems $2 \& 3$ is the same in each case and is explained in $\S 2$. Given a curve $Z \subset \mathbb{P}^{2}$, the idea is to conjugate $\tau \in \operatorname{Dec}(Z)$ to $\tau^{\prime} \in \operatorname{Dec}(Y)$, for a curve $Y \subset \mathbb{P}^{2}$ of lower degree, and then use the result for $Y$.

Remark 1.3. The proof of each theorem is elementary and only requires choosing quadratic transformations with base points that lie outside of a collection of finitely many points and lines. In the cubic case we need to choose base points which avoid all of the tangent lines to a conic which pass through a given point. We must restrict to a field $\mathrm{k}$ of characteristic $\neq 2$ in this case, since over fields of characteristic 2 every line through a given point may be tangent to a conic (see [12, Appendix to $\S 2]$ ).

Remark 1.4. As shown in Proposition 3.5, for a conic $C$ it is still possible to write $\operatorname{Dec}(C)=\left\langle\operatorname{Aut}\left(\mathbb{P}^{2}, C\right), \sigma\right\rangle$ using just one suitably general elementary quadratic transformation $\sigma$ (where 'suitably general' means that $\sigma$ does not contract a tangent line to $C$ ). However, if the base field $\mathrm{k}$ is uncountable then we need an uncountable number of elementary quadratic transformations to generate both Ine $(C)$ (see Remark 3.6) and $\operatorname{Dec}(X)$ for $X$ a nodal cubic (see $\S 4.3)$.

1.4. Acknowledgements. We would like to thank Eric Bedford and Jeffrey Diller for helpful comments.

\section{The main Proposition}

Let $Y, Z \subset \mathbb{P}^{2}$ be two arbitrary irreducible plane curves.

Definition 2.1. Let $\Phi_{Y, Z} \subset \operatorname{Bir}\left(\mathbb{P}^{2}\right)$ be the set of all elementary quadratic transformations $\varphi$ which map $Y$ birationally onto $Z$.

Note that $\Phi_{Y, Z}$ is a (possibly empty) subset of $\operatorname{Bir}\left(\mathbb{P}^{2}\right)$ and not a subgroup. For any $\varphi, \psi \in \Phi_{Y, Z}$ we clearly have $\varphi \psi^{-1} \in \operatorname{Dec}(Z)$. More generally for any $\tau \in \operatorname{Dec}(Y)$ we have $\varphi \tau \psi^{-1} \in \operatorname{Dec}(Z)$.

Proposition 2.2. Suppose that $\Phi_{Y, Z} \neq \emptyset$ and the following three statements hold:

(A) Any $\tau \in \operatorname{Dec}(Y)$ can be factored into elementary quadratic transformations inside $\operatorname{Dec}(Y)$.

(B) For any $\varphi, \psi \in \Phi_{Y, Z}$ the composition $\varphi \psi^{-1} \in \operatorname{Dec}(Z)$ can be factored into elementary quadratic transformations inside $\operatorname{Dec}(Z)$.

(C) For any elementary quadratic transformation $\tau \in \operatorname{Dec}(Y)$ there exist $\varphi, \psi \in \Phi_{Y, Z}$ such that $\varphi \tau \psi^{-1} \in \operatorname{Dec}(Z)$ can be factored into elementary quadratic transformations inside $\operatorname{Dec}(Z)$.

Then any $\tau \in \operatorname{Dec}(Z)$ can be factored into elementary quadratic transformations inside $\operatorname{Dec}(Z)$. 
Proof. Suppose that $\tau \in \operatorname{Dec}(Z)$ and choose any two maps $\varphi, \psi \in \Phi_{Y, Z} \neq \emptyset$. Then by (A) we can factor $\tau^{\prime}:=\psi^{-1} \tau \varphi \in \operatorname{Dec}(Y)$ into elementary quadratic transformations $\tau^{\prime}=\tau_{n} \tau_{n-1} \cdots \tau_{2} \tau_{1}$ with $\tau_{i} \in \operatorname{Dec}(Y)$ for all $i=1, \ldots, n$.

By $(\mathrm{C})$ we can find $\varphi_{i}, \psi_{i} \in \Phi_{Y, Z}$ such that $f_{i}:=\varphi_{i} \tau_{i} \psi_{i}^{-1} \in \operatorname{Dec}(Z)$ can be factored into elementary quadratic transformations inside $\operatorname{Dec}(Z)$ for all $i=1, \ldots, n$.

Now let $\varphi_{0}:=\varphi$ and $\psi_{n+1}:=\psi$. Then by (B) we can factor $g_{i}:=\psi_{i+1} \varphi_{i}^{-1} \in \operatorname{Dec}(Z)$ into elementary quadratic transformations inside $\operatorname{Dec}(Z)$ for all $i=0, \ldots, n$.

We can write $\tau=g_{n} f_{n} g_{n-1} \cdots g_{1} f_{1} g_{0}$, according to the diagram:

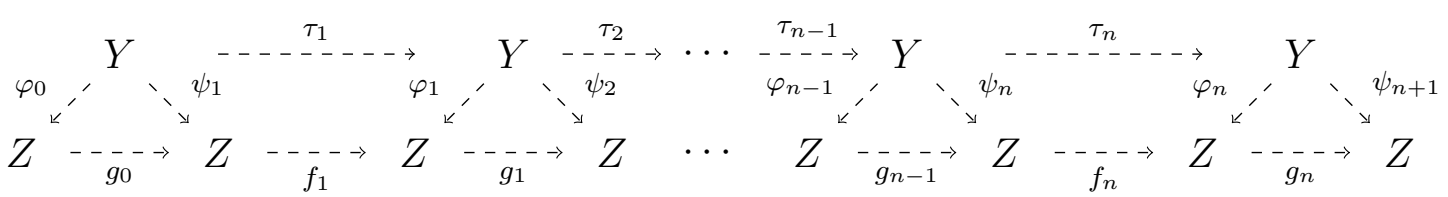

and hence we can factor $\tau$ into elementary quadratic transformations inside $\operatorname{Dec}(Z)$.

Theorem 2 and Theorem 3 follow from Proposition 2.2, where the three statements (A), (B), (C) appearing in the proposition are proved in each case according to:

\begin{tabular}{c|ccc} 
& $(\mathrm{A})$ & $(\mathrm{B})$ & $(\mathrm{C})$ \\
\hline Theorem 2 & Theorem 1 & Lemma 3.2 & Lemma 3.3 \\
Theorem 3 & Theorem 2 & Lemma 4.2 & Lemma 4.3
\end{tabular}

\section{The DeComposition group of A CONIC}

Throughout this section we let $L \subset \mathbb{P}^{2}$ denote a fixed line and $C \subset \mathbb{P}^{2}$ a conic.

Remark 3.1. If $\varphi \in \operatorname{Bir}\left(\mathbb{P}^{2}\right)$ is an elementary quadratic transformation belonging to $\Phi_{L, C}$ then all three base points of $\varphi$ must lie outside of $L$. Conversely, given any three non-collinear points in $\mathbb{P}^{2} \backslash L$ we can always find an elementary quadratic transformation $\varphi \in \Phi_{L, C}$ with these as base points.

3.1. Proof of Theorem 2. We prove statements (B) \& (C) in Proposition 2.2 in the special case that $Y=L$ a line and $Z=C$ a conic.

\subsubsection{Proof of statement (B) for conics.}

Lemma 3.2. Suppose that $\varphi_{1}, \varphi_{2} \in \Phi_{L, C}$. Then the composition $\varphi_{2} \varphi_{1}^{-1} \in \operatorname{Dec}(C)$ can be factored into elementary quadratic transformations inside $\operatorname{Dec}(C)$.

Proof. For $i=1,2$, we let $P_{i}, Q_{i}, R_{i}$ be the base points of $\varphi_{i}$, none of which lie on $L$. We may assume that these six points are in general position, i.e. that no points coincide and that no three points are collinear, as in Figure 1(i). If this is not the case, choose a third map $\varphi_{3} \in \Phi_{L, C}$ whose base points are in general position with respect to both $\varphi_{1}$ and $\varphi_{2}$. Then we can write $\varphi_{2} \varphi_{1}^{-1}=\left(\varphi_{2} \varphi_{3}^{-1}\right)\left(\varphi_{3} \varphi_{1}^{-1}\right)$ and decompose each of $\varphi_{2} \varphi_{3}^{-1}$ and $\varphi_{3} \varphi_{1}^{-1}$ into elementary quadratic transformations inside $\operatorname{Dec}(C)$. 
We let $\varphi_{1}=: \psi_{0}, \psi_{1}, \psi_{2}, \psi_{3}:=\varphi_{2} \in \Phi_{L, C}$ be a sequence of elementary quadratic transformations with base points:

$$
\left(P_{1}, Q_{1}, R_{1}\right),\left(P_{1}, Q_{1}, R_{2}\right),\left(P_{1}, Q_{2}, R_{2}\right),\left(P_{2}, Q_{2}, R_{2}\right)
$$

and we write $\varphi_{2} \varphi_{1}^{-1}=\left(\psi_{3} \psi_{2}^{-1}\right)\left(\psi_{2} \psi_{1}^{-1}\right)\left(\psi_{1} \psi_{0}^{-1}\right)$.

By our assumption, $\psi_{1}$ and $\psi_{2}$ exist since no three points are collinear and we can take $\psi_{1}, \psi_{2} \in \Phi_{L, C}$ since none of these points lie on $L$. Moreover $\psi_{i+1} \psi_{i}^{-1} \in \operatorname{Dec}(C)$ is an elementary quadratic transformation for $i=0,1,2$ since $\psi_{i}$ and $\psi_{i+1}$ share exactly two common base points and no three base points are collinear.

(i)

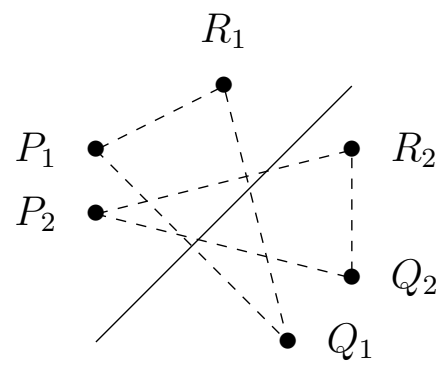

(ii)

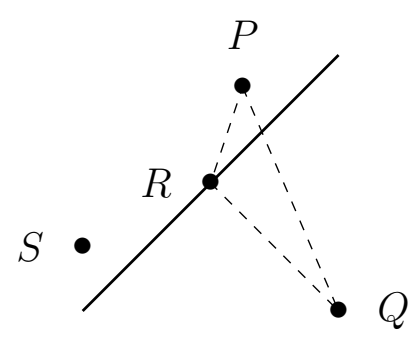

Figure 1. Configuration of base points in proof of (i) Lemma 3.2 and (ii) Lemma 3.3. 3.1.2. Proof of statement (C) for conics. In fact we prove a stronger statement than statement $(\mathrm{C})$ (since $\mathrm{id}_{\mathbb{P}^{2}}$ is a decomposition into zero elementary quadratic transformations in $\operatorname{Dec}(C))$.

Lemma 3.3. Let $\tau \in \operatorname{Dec}(L)$ be an elementary quadratic transformation. Then we can find $\varphi, \psi \in \Phi_{L, C}$ such that $\varphi \tau \psi^{-1}=\mathrm{id}_{\mathbb{P}^{2}}$.

Proof. Let $P, Q, R$ be the base points of $\tau$, where $P, Q \notin L$ and $R \in L$. Choose a point $S \notin L$ as in Figure 1(ii), such that no three of $P, Q, R, S$ are collinear.

Since $P, Q, S$ are non-collinear we let $\psi \in \Phi_{L, C}$ be an elementary quadratic transformation with these base points. Then $\varphi:=\psi \tau^{-1} \in \Phi_{L, C}$ is also an elementary quadratic transformation since $\psi$ and $\tau$ share two base points and no three base points are collinear. Thus $\varphi \tau \psi^{-1}=\mathrm{id}_{\mathbb{P}^{2}}$.

3.2. A generating set for $\operatorname{Dec}(C)$. It was shown in [10] that, for $L \subset \mathbb{P}^{2}$ a line, $\operatorname{Dec}(L)$ can be generated by $\operatorname{Aut}\left(\mathbb{P}^{2}, L\right)$ and any one elementary quadratic transformation $\sigma \in \operatorname{Dec}(L)$. This is because $\operatorname{Aut}\left(\mathbb{P}^{2}, L\right)$ is still large enough to act transitively on the set:

$$
B=\left\{(P, Q, R) \in\left(\mathbb{P}^{2}\right)^{3} \mid P \in L \text { and } Q, R \notin L \text { non-collinear }\right\}
$$

of all possible base points for $\sigma$. For the conic $C \subset \mathbb{P}^{2}$, even though the analogous action of $\operatorname{Aut}\left(\mathbb{P}^{2}, C\right)$ is no longer transitive, it is still true that $\operatorname{Dec}(C)$ can be generated by $\operatorname{Aut}\left(\mathbb{P}^{2}, C\right)$ and a suitably general elementary quadratic transformation $\sigma \in$ $\operatorname{Dec}(C)$.

We fix a model $C=V\left(x z-y^{2}\right) \subset \mathbb{P}^{2}$ in order to describe $\operatorname{Aut}\left(\mathbb{P}^{2}, C\right)$.

Lemma 3.4. Aut $\left(\mathbb{P}^{2}, C\right)$ is given by:

$$
\operatorname{Aut}\left(\mathbb{P}^{2}, C\right)=\left\{\left(\begin{array}{ccc}
a^{2} & 2 a b & b^{2} \\
a c & a d+b c & b d \\
c^{2} & 2 c d & d^{2}
\end{array}\right) \in \mathrm{PGL}_{3} \mid a d-b c \neq 0\right\} \simeq \mathrm{PGL}_{2} .
$$

In particular any $\alpha \in \mathrm{PGL}_{2}=\operatorname{Bir}(C)$ extends uniquely to a linear map in $\operatorname{Aut}\left(\mathbb{P}^{2}, C\right)$. 
It follows from Lemma 3.4 that $\operatorname{Ine}(C) \cap \mathrm{PGL}_{3}=\left\langle\mathrm{id}_{\mathbb{P}^{2}}\right\rangle$. Moreover the sequence

$$
1 \rightarrow \operatorname{Ine}(C) \rightarrow \operatorname{Dec}(C) \rightarrow \mathrm{PGL}_{2} \rightarrow 1
$$

is exact and $\operatorname{Dec}(C)=\operatorname{Ine}(C) \rtimes \mathrm{PGL}_{2}$ is a semidirect product, where $\mathrm{PGL}_{2}$ acts on Ine $(C)$ by conjugation.

Proposition 3.5. $\operatorname{Dec}(C)=\left\langle\operatorname{Aut}\left(\mathbb{P}^{2}, C\right), \sigma\right\rangle$ for any elementary quadratic transformation $\sigma$ which does not contract a tangent line to $C$.

Proof. Let $\tau \in \operatorname{Dec}(C)$ be an elementary quadratic transformation and consider the action of $\mathrm{PGL}_{2} \simeq \operatorname{Aut}\left(\mathbb{P}^{2}, C\right)$ on the set:

$$
B=\left\{(P, Q, R) \in\left(\mathbb{P}^{2}\right)^{3} \mid P, Q \in C \text { and } R \notin C \text { non-collinear }\right\}
$$

of all possible base points for $\tau$. If $P, Q \in C$ and $R \notin C$ are the (ordered) base points of $\tau$ then, by an element of $\mathrm{PGL}_{2}$, we can send $P \mapsto(1: 0: 0), Q \mapsto(0: 0: 1)$ and $R$ to a point in the conic $\Gamma_{d}=V\left(x z-d y^{2}\right)$ for a uniquely determined $1 \neq d \in \mathrm{k}$. Write $B=\bigcup_{d \in \mathrm{k} \backslash 1} B_{d}$, a decomposition into $\mathrm{PGL}_{2}$-invariant sets according to this pencil of conics $\Gamma_{d}$. The sets $B_{d}$ with $d \neq 0$ are all $\mathrm{PGL}_{2}$-orbits. For the degenerate conic $\Gamma_{0}$ the set $B_{0}$ splits into three $\mathrm{PGL}_{2}$-orbits $B_{0}=B_{1,0} \cup B_{0,1} \cup B_{0,0}$ according to the cases:

$$
R \in \Gamma_{1,0}:=\{(t: 1: 0) \mid t \neq 0\}, \quad R \in \Gamma_{0,1}:=\{(0: 1: t) \mid t \neq 0\}, \quad R=(0: 1: 0) .
$$

As shown in Figure 2, these three orbits correspond to the cases where one or two of the lines contracted by $\tau$ are tangent to $C$.

(i)

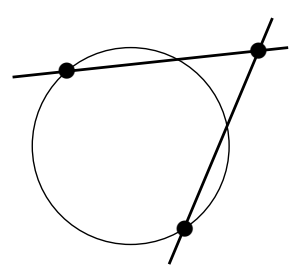

(ii)

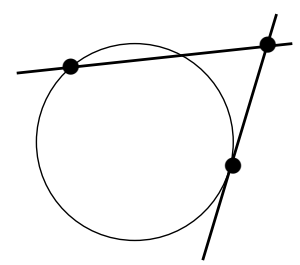

(iii)

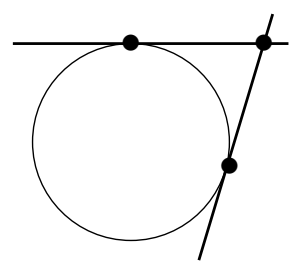

Figure 2. The base points of $\tau$ belonging to the orbit (i) $B_{d}$ with $d \neq 0$, (ii) $B_{1,0}$ or $B_{0,1}$, (iii) $B_{0,0}$.

Let $\sigma_{a, b} \in \operatorname{Dec}(C)$ be an elementary quadratic transformation with base points $(1: 0: 0)$, $(0: 0: 1)$ and $(a: 1: b)$ belonging to an orbit $B_{a b}$ with $a b \neq 0$. By composing with a suitable linear map we can assume the map is actually in $\operatorname{Ine}(C)$, in which case $\sigma_{a, b}$ is uniquely determined and given by:

$$
\sigma_{a, b}=\left((1-a b) x y+a\left(x z-y^{2}\right): x z-a b y^{2}:(1-a b) y z+b\left(x z-y^{2}\right)\right) .
$$

Any elementary quadratic transformation $\sigma \in \operatorname{Dec}(C)$ which does not contract a tangent line to $C$ has base points belonging to the same $\mathrm{PGL}_{2}$-orbit as $\sigma_{a, b}$ for some $a, b \in \mathrm{k}$ with $a b \neq 0,1$. Therefore, to prove the proposition, it is enough to show that given any $a, b \in \mathrm{k}$ with $a b \neq 0,1$, we can use $\sigma_{a, b}$ to generate at least one elementary quadratic transformation with base points belonging to any other $\mathrm{PGL}_{2}$-orbit.

Consider the linear map:

$$
\lambda_{a, b}=\left(x+2 a y+a^{2} z: b x+(1+a b) y+a z: b^{2} x+2 b y+z\right)
$$

and, for $c \neq 0,1, \infty$, the diagonal map $\mu_{c}=\left(c^{2} x: c y: z\right)$. Since $a b \neq 0$ we get the formula:

$$
\sigma_{a^{\prime}, b^{\prime}}=\lambda_{a, b}^{-1} \mu_{c}^{-1} \sigma_{a, b} \mu_{c} \sigma_{a, b}^{-1} \lambda_{a, b}
$$


where $a^{\prime}=\frac{1-a b c}{b(c-1)}$ and $b^{\prime}=\frac{a b-c}{a(c-1)}$.

As $c$ varies the base points of $\sigma_{a^{\prime}, b^{\prime}}$ are $(1: 0: 0),(0: 0: 1)$ and the point $R^{\prime}=$ $(a(1-a b c): a b(c-1): b(a b-c))$ lying on the line:

$$
L_{a, b}=V(b x+(1+a b) y+a z) .
$$

The point $R^{\prime}$ can be any point on $L_{a, b}$, except for $(a: 0:-b)$, corresponding to $c=1$, and $L_{a, b} \cap C=\left\{\left(-\frac{1}{b}: 1:-b\right),\left(-a: 1:-\frac{1}{a}\right)\right\}$, corresponding to $c=0, \infty$. Outside of these points $L_{a, b}$ intersects every conic $\Gamma_{d}$ at least once.

For all $d \neq 0$ this construction gives an elementary quadratic transformation with base points in $B_{d}$.

If $d=0$ and $a b \neq-1$ then $L_{a, b}$ meets $\Gamma_{1,0}$ and $\Gamma_{0,1}$ giving elementary quadratic transformations with base points in $B_{1,0}$ and $B_{0,1}$. If $a b=-1$ then $L_{a, b} \cap \Gamma_{0}=(0: 1: 0)$ giving an elementary quadratic transformation with base points in $B_{0,0}$.

It remains to produce an elementary quadratic transformation with base points in $B_{0,0}$ if $a b \neq-1$ and in $B_{1,0}$ and $B_{0,1}$ if $a b=-1$. We can use the construction once to produce $\sigma_{a^{\prime}, b^{\prime}}$ with $a^{\prime} b^{\prime}=-1$ if $a b \neq-1$ (or with $a^{\prime} b^{\prime} \neq-1$ if $a b=-1$ ) and then proceed as above.

Remark 3.6. If the ground field $\mathrm{k}$ is uncountable then the corresponding statement for Ine $(C)$ is not true, i.e. Ine $(C)$ cannot be generated by linear maps and any countable collection of elementary quadratic maps. Although Ine $(C) \cap \mathrm{PGL}_{3}$ is trivial, Ine $(C)$ contains a lot of elementary quadratic transformations. Indeed the maps

$$
\left\{\sigma_{a, b} \in \operatorname{Ine}(C) \mid a, b \in \mathrm{k}, a b \neq 1\right\}
$$

appearing in the proof of Proposition 3.5 give an uncountable family.

\section{The DeComposition Group of A RATional CUBiC}

Throughout this section we let $C \subset \mathbb{P}^{2}$ denote a fixed conic and $X \subset \mathbb{P}^{2}$ a rational cubic. We will distinguish between the nodal and cuspidal cases when necessary. As explained in Remark 1.3, we will also assume that the characteristic of $\mathrm{k}$ is not 2.

Remark 4.1. Any map $\varphi \in \Phi_{C, X}$ must have exactly one base point $P \in C$ and two base points $Q, R \notin C$. In this case $X$ is a cuspidal cubic if the line $\overline{Q R}$ is tangent to $C$ and a nodal cubic otherwise, as shown in Figure 3. Moreover, given any three noncollinear points in such a position we can always find a map $\varphi \in \Phi_{C, X}$ with these three points as base points.

(i)

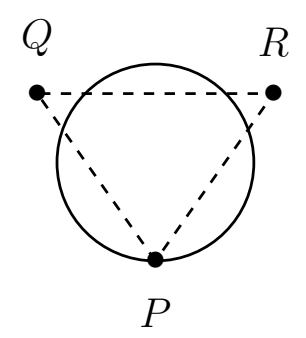

(ii)

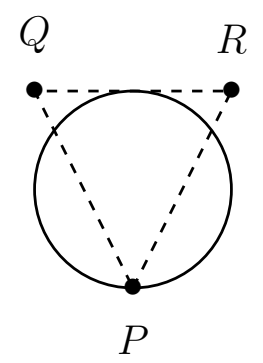

Figure 3. Base point configurations for $\varphi \in \Phi_{C, X}$ when $X$ is (i) a nodal cubic and (ii) a cuspidal cubic. 
4.1. Proof of Theorem 3. We now prove statements (B) \& (C) in Proposition 2.2 for $Y=C$ a conic and $Z=X$ a rational cubic.

\subsubsection{Proof of statement (B) for cubics.}

Lemma 4.2. Suppose that $\varphi_{1}, \varphi_{2} \in \Phi_{C, X}$. Then the composition $\varphi_{2} \varphi_{1}^{-1} \in \operatorname{Dec}(X)$ can be factored into elementary quadratic transformations inside $\operatorname{Dec}(X)$.

Proof. For $i=1,2$, we let $P_{i}, Q_{i}, R_{i}$ be the base points of $\varphi_{i}$, where $P_{i} \in C$ and $Q_{i}, R_{i} \notin C$. As in the proof of Lemma 3.2, we may intertwine with a third map $\varphi_{3} \in \Phi_{C, X}$ to assume that no base points coincide, no three are collinear and no two lie on a tangent line to $C$ (unless $X$ is a cuspidal cubic, in which case we can assume that only $Q_{1}, R_{1}$ and $Q_{2}, R_{2}$ lie on a tangent line to $\left.C\right)$.

The nodal case: If $X$ is a nodal cubic we let $\varphi_{1}=: \psi_{0}, \psi_{1}, \psi_{2}, \psi_{3}:=\varphi_{2} \in \Phi_{C, X}$ be a sequence of elementary quadratic transformations with base points:

$$
\left(P_{1}, Q_{1}, R_{1}\right),\left(P_{1}, Q_{1}, R_{2}\right),\left(P_{1}, Q_{2}, R_{2}\right),\left(P_{2}, Q_{2}, R_{2}\right)
$$

and we write $\varphi_{2} \varphi_{1}^{-1}=\left(\psi_{3} \psi_{2}^{-1}\right)\left(\psi_{2} \psi_{1}^{-1}\right)\left(\psi_{1} \psi_{0}^{-1}\right)$.

By our assumption $\psi_{1}$ and $\psi_{2}$ exist since each of these triples is non-collinear and $\psi_{1}, \psi_{2} \in \Phi_{C, X}$ since they both have precisely one base point on $C$ and do not contract any tangent line to $C$. Lastly each composition $\psi_{i+1} \psi_{i}^{-1} \in \operatorname{Dec}(X)$ is an elementary quadratic transformation since $\psi_{i}$ and $\psi_{i+1}$ share exactly two common base points and no three base points are collinear.

(i)

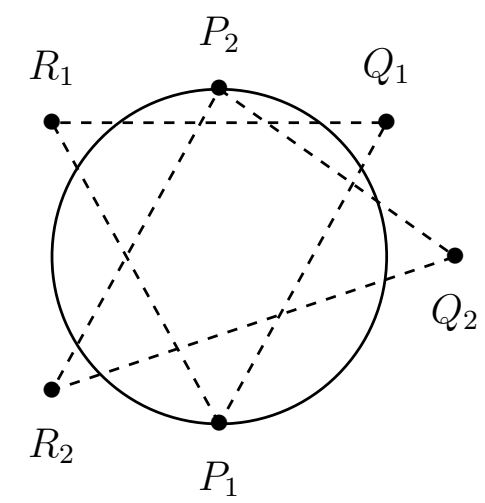

(ii)

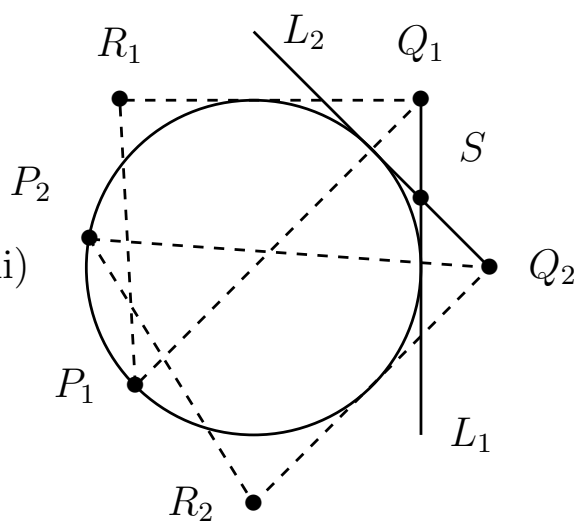

Figure 4. Configuration of base points in (i) the nodal case and (ii) the cuspidal case.

The cuspidal case: If $X$ is a cuspidal cubic then we must be a little bit more careful to ensure that each of our intermediate maps $\psi_{i}$ contracts a tangent line to $C$.

For $i=1,2$ let $L_{i}$ be the tangent line to $C$ passing through $Q_{i}$ which does not contain $R_{i}$. By our assumption on the position of the base points, the point $S=L_{1} \cap L_{2}$ is well-defined, $S \notin C$ and $S$ is not equal to any $P_{i}, Q_{i}, R_{i}$. Moreover, no three of the seven points $P_{1}, P_{2}, Q_{1}, Q_{2}, R_{1}, R_{2}, S$ are collinear.

Now we let $\varphi_{1}=: \psi_{0}, \psi_{1}, \psi_{2}, \psi_{3}, \psi_{4}:=\varphi_{2} \in \Phi_{C, X}$ be a sequence of elementary quadratic transformations with base points:

$$
\left(P_{1}, Q_{1}, R_{1}\right),\left(P_{1}, Q_{1}, S\right),\left(P_{1}, Q_{2}, S\right),\left(P_{2}, Q_{2}, S\right),\left(P_{2}, Q_{2}, R_{2}\right)
$$

and we write $\varphi_{2} \varphi_{1}^{-1}=\left(\psi_{4} \psi_{3}^{-1}\right)\left(\psi_{3} \psi_{2}^{-1}\right)\left(\psi_{2} \psi_{1}^{-1}\right)\left(\psi_{1} \psi_{0}^{-1}\right)$. 
As before, $\psi_{1}, \psi_{2}, \psi_{3}$ exist since each triple of base points is non-collinear and $\psi_{1}, \psi_{2}, \psi_{3} \in$ $\Phi_{C, X}$ since they all have precisely one base point on $C$ and contract a tangent line to $C$. Lastly each composition $\psi_{i+1} \psi_{i}^{-1} \in \operatorname{Dec}(X)$ is an elementary quadratic transformation since $\psi_{i}, \psi_{i+1}$ share exactly two common base points and no three base points are collinear.

\subsubsection{Proof of statement (C) for cubics.}

Lemma 4.3. Let $\tau \in \operatorname{Dec}(C)$ be an elementary quadratic transformation. Then we can find $\varphi, \psi \in \Phi_{C, X}$ such that $\varphi \tau \psi^{-1} \in \operatorname{Dec}(X)$ can be factored into elementary quadratic transformations inside $\operatorname{Dec}(X)$.

Proof. We first assume that $\tau$ is an elementary quadratic transformations which does not contract a tangent line to $C$ (i.e. $\tau$ has a configuration of base points as in Figure 2(i)). Let $P, Q \in C$ and $R \notin C$ be the base points of $\tau$ and let $L$ be a tangent line to $C$ passing through $R$. By assumption $L \neq \overline{P R}, \overline{Q R}$.

Choose a point $S \notin C$ as in Figure 5 , such that no three of $P, Q, R, S$ are collinear. If $X$ is a nodal cubic then we choose $S$ to avoid the tangent lines to $C$ passing through $P, Q$ or $R$. If $X$ is a cuspidal cubic then we choose $S$ to lie on $L$ but avoid the tangent lines to $C$ through $P$ or $Q$.

(i)

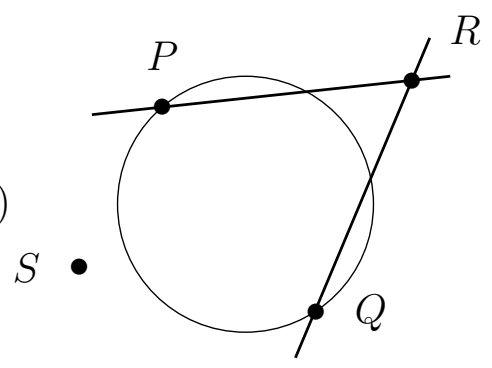

(ii)

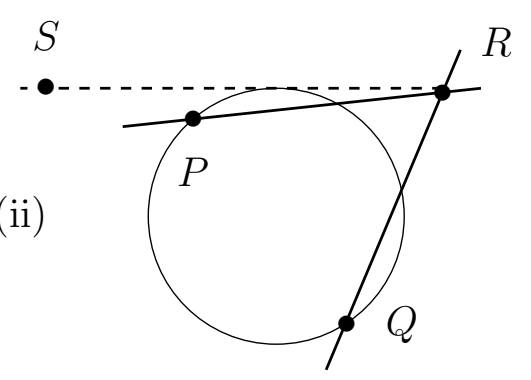

FiguRE 5. Location of the point $S$ when $X$ is (i) a nodal cubic and (ii) a cuspidal cubic.

Since $P, R, S$ are non-collinear there is an elementary quadratic transformation $\psi \in \Phi_{C, X}$ with these base points. We let $\varphi:=\psi \tau^{-1} \in \Phi_{C, X}$ which is also an elementary quadratic transformation since $\psi$ and $\tau$ share two base points and no three of the base points are collinear. Thus $\varphi \tau \psi^{-1}=\operatorname{id}_{\mathbb{P}^{2}} \in \operatorname{Dec}(X)$ which is a decomposition into zero elementary quadratic transformations inside $\operatorname{Dec}(X)$.

If $\tau$ is an arbitrary elementary quadratic transformation, then by Proposition 3.5 we can write $\tau=\tau_{n} \cdots \tau_{1}$ where $\tau_{i} \in \operatorname{Dec}(C)$ are elementary quadratic transformations which do not contract a tangent line to $C$. We can find $\varphi_{i}, \psi_{i} \in \Phi_{C, X}$, for $i=1, \ldots, n$, such that $\varphi_{i} \tau_{i} \psi_{i}^{-1} \in \operatorname{Dec}(X)$ can be factored into elementary quadratic transformations inside $\operatorname{Dec}(X)$ and by Lemma 4.2 we can factor $\psi_{i+1} \varphi_{i}^{-1} \in \operatorname{Dec}(X)$ into elementary quadratic transformations inside $\operatorname{Dec}(X)$ for $i=1, \ldots, n-1$. Therefore, taking $\varphi:=\varphi_{n}$ and $\psi:=\psi_{1}$, we can factor

$$
\varphi \tau \psi^{-1}=\left(\varphi_{n} \tau_{n} \psi_{n}^{-1}\right)\left(\psi_{n} \varphi_{n-1}^{-1}\right)\left(\varphi_{n-1} \tau_{n-1} \psi_{n-1}^{-1}\right) \cdots\left(\psi_{2} \varphi_{1}^{-1}\right)\left(\varphi_{1} \tau_{1} \psi_{1}^{-1}\right)
$$

into elementary quadratic transformations inside $\operatorname{Dec}(X)$. 
4.2. An example. Let $X$ be a nodal (resp. cuspidal) cubic, let $\tau \in \operatorname{Dec}(X)$ and suppose that we conjugate $\tau$ to get $\tau^{\prime} \in \operatorname{Dec}(C)$, for a conic $C$, as in the proof of Proposition 2.2. If $\tau^{\prime}$ can be decomposed into $n$ elementary quadratic transformations which do not contract any tangent line to $C$ then naïvely applying the proof of Theorem 3 gives a decomposition of $\tau$ into at most $6(n+1)($ resp. $8(n+1))$ elementary quadratic transformations inside $\operatorname{Dec}(X)$.

Even in relatively simple cases this gives a very long decomposition which is far from optimal. For example let $X$ be the cuspidal cubic $X=V\left(x^{3}-y^{2} z\right) \subset \mathbb{P}^{2}$ and consider the de Jonquières involution $\tau=\left(x y^{2}: y^{3}: 2 x^{3}-y^{2} z\right) \in \operatorname{Ine}(X)$. This map has one proper base point at the cusp point $P \in X$ and all other base points infinitely near to $P$. If $C$ is the conic $C=V\left(x z-y^{2}\right)$ then $\varphi=(x(y+z): x(x+y): z(y+z)) \in \Phi_{C, X}$ and conjugating $\tau$ with $\varphi$ gives $\tau^{\prime}=\varphi^{-1} \tau \varphi \in \operatorname{Dec}(C)$, a map of degree 3 with two proper base points, which decomposes into four elementary quadratic transformations in $\operatorname{Dec}(C)$ not contracting any tangent line to $C$. Therefore we can decompose $\tau$ into at worst 40 elementary quadratic transformations inside $\operatorname{Dec}(X)$, although we expect a minimal decomposition to be much shorter.

4.3. Generating sets for $\operatorname{Dec}(X)$. Let $X$ be the nodal cubic given by the model $X=V\left(x^{3}+y^{3}-x y z\right) \subset \mathbb{P}^{2}$. We see that $\operatorname{Aut}\left(\mathbb{P}^{2}, X\right)$ is the finite group given by:

$$
\operatorname{Aut}\left(\mathbb{P}^{2}, X\right)=\left\langle\left(\begin{array}{ccc}
\omega & 0 & 0 \\
0 & \omega^{2} & 0 \\
0 & 0 & 1
\end{array}\right),\left(\begin{array}{lll}
0 & 1 & 0 \\
1 & 0 & 0 \\
0 & 0 & 1
\end{array}\right)\right\rangle \simeq S_{3}
$$

where $\omega \in \mathrm{k}$ is a primitive cube root of unity. If $\mathrm{k}$ is an uncountable field then $\operatorname{Dec}(X)$ is an uncountable group and therefore cannot be generated by $\operatorname{Aut}\left(\mathbb{P}^{2}, X\right)$ and any finite (or countable) collection of elementary quadratic transformations.

Now suppose $X$ is the cuspidal cubic given by the model $X=V\left(x^{3}-y^{2} z\right) \subset \mathbb{P}^{2}$. In this case $\operatorname{Aut}\left(\mathbb{P}^{2}, X\right)$ is infinite:

$$
\operatorname{Aut}\left(\mathbb{P}^{2}, X\right)=\left\langle\left(\begin{array}{ccc}
a & 0 & 0 \\
0 & 1 & 0 \\
0 & 0 & a^{3}
\end{array}\right) \mid a \in \mathrm{k}^{\times}\right\rangle \simeq \mathbb{G}_{m} .
$$

We do not know whether or not $\operatorname{Dec}(X)$ can be generated by $\operatorname{Aut}\left(\mathbb{P}^{2}, X\right)$ and any countable collection of elementary quadratic transformations.

\section{RAtional CURVES OF HigheR DEGREE}

We provide a family of plane rational curves $X_{d} \subset \mathbb{P}^{2}$, birationally equivalent to a line and of degree $d \geq 4$, to show that we cannot expect Theorems $1,2 \& 3$ to be true for curves of higher degree.

Let $X_{d}$ denote the rational curve given by $X_{d}=V\left(x^{d}-y^{d-1} z\right) \subset \mathbb{P}^{2}$ which has a unique singular point $P=(0: 0: 1)$, a cusp of multiplicity $d-1$, and a unique inflection point $Q=(0: 1: 0)$. Let $L_{Q}=(z=0)$ be the tangent line intersecting $X_{d}$ at $Q$ with multiplicity $d$ and let $L_{P}=(y=0)$ be the tangent line to the cusp $P$. Any de Jonquières transformation of degree $d$ with major base point at $P$ and all other base points on $X_{d} \backslash P$ sends $X_{d}$ onto a line.

A map in $\operatorname{Aut}\left(\mathbb{P}^{2}, X_{d}\right)$ has to fix $P$ and $Q$ and preserve $L_{P}$ and $L_{Q}$. It is straightforward to check that:

$$
\operatorname{Aut}\left(\mathbb{P}^{2}, X_{d}\right)=\left\{\left(a x: y: a^{d} z\right) \mid a \in \mathrm{k}^{\times}\right\} \simeq \mathbb{G}_{m} .
$$


Lemma 5.1. The standard involution $\sigma=(y z: z x: x y) \in \operatorname{Bir}\left(\mathbb{P}^{2}\right)$ is the only elementary quadratic map that preserves $X_{d}$, up to composition with an element of $\operatorname{Aut}\left(\mathbb{P}^{2}, X_{d}\right)$.

Proof. It is easy to check that $\sigma \in \operatorname{Dec}\left(X_{d}\right)$. Any other elementary quadratic transformation $\tau \in \operatorname{Dec}\left(X_{d}\right)$ must have one base point at $P \in X_{d}$, one base point in the smooth locus of $X_{d}$ and one base point not contained in $X_{d}$. In particular $\tau^{-1}$ also has a base point at $P$. Since the line $\tau^{-1}(P)$ is tangent to a point of $X_{d}$ with multiplicity $\geq d-1$, we must have $\tau^{-1}(P)=L_{Q}$. As the line $L_{Q}$ is contracted, both $\tau$ and $\tau^{-1}$ must have two base points on $L_{Q}$, one of which is $L_{Q} \cap X_{d}=Q$. Now the line $\tau^{-1}(Q)$ is tangent to the cusp $P$ so we must have $\tau^{-1}(Q)=L_{P}$, as in Figure 6 .

Since the lines $L_{P}$ and $L_{Q}$ are contracted, the base points of $\tau$ are $P=(0: 0: 1)$, $Q=(0: 1: 0)$ and $L_{P} \cap L_{Q}=(1: 0: 0)$. Hence, up to an element of $\operatorname{Aut}\left(\mathbb{P}^{2}, X_{d}\right)$, we must have $\tau=\sigma$.
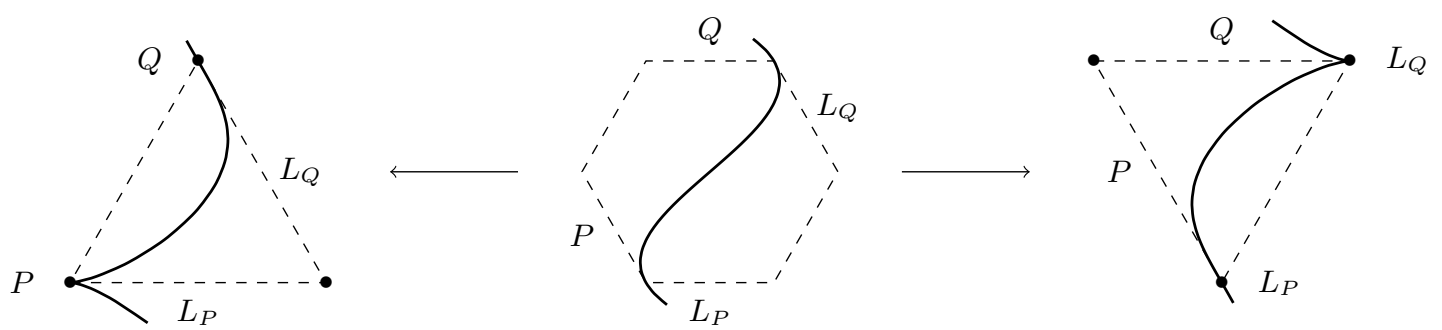

FiguRE 6. Resolution of the standard involution $\sigma \in \operatorname{Dec}\left(X_{d}\right)$.

Proposition 5.2. If $d \geq 4$, the group Dec $\left(X_{d}\right)$ cannot be generated by linear maps and elementary quadratic transformations.

Proof. By Lemma 5.1, the subgroup of $\operatorname{Dec}\left(X_{d}\right)$ generated by linear maps and elementary quadratic transformations is given by $\left\langle\operatorname{Aut}\left(\mathbb{P}^{2}, X_{d}\right), \sigma\right\rangle$. Since $\sigma^{2}=\mathrm{id}_{\mathbb{P}^{2}}$ and $\sigma \lambda=\lambda^{-1} \sigma$ for any $\lambda \in \operatorname{Aut}\left(\mathbb{P}^{2}, X_{d}\right)$, all elements of this subgroup are of the form $\lambda$ or $\lambda \sigma$ and are either linear or quadratic. But there are many elements in $\operatorname{Dec}\left(X_{d}\right)$ of degree $>2$; for example the de Jonquières transformation $\tau_{a}=\left(x y^{d-1}: y^{d}:(1-a) x^{d}+a y^{d-1} z\right)$ for $a \in \mathrm{k}^{\times}$.

Remark 5.3. The family of maps $\left\{\tau_{a} \mid a \in \mathrm{k}^{\times}\right\}$, appearing at the end of the proof of Proposition 5.2, form a subgroup of Ine $\left(X_{d}\right)$ isomorphic to $\mathbb{G}_{m}$ since $\tau_{b} \tau_{a}=\tau_{a b}$ for all $a, b \in \mathrm{k}^{\times}$.

\section{REFERENCES}

[1] E. Bedford, K. Kim Dynamics of Rational Surface Automorphisms: Linear Fractional Recurrences, J. Geom. Anal., 19 (2009), 553-583.

[2] J. Blanc On the inertia group of elliptic curves in the Cremona group of the plane, Michigan Math. J., 56, no. 2 (2008), 315-330.

[3] J. Blanc, I. Pan, T. Vust Sur un théorème de Castelnuovo Bull. Braz. Math. Soc. (N.S.), 39, no. 1 (2008), 61-80.

[4] J. Blanc, I. Pan, T. Vust On birational transformations of pairs in the complex plane Geom. Dedicata, 139 (2009), 57-73.

[5] S. CAntat, S. LAMY Normal subgroups in the Cremona group, With an appendix by Yves de Cornulier, Acta Math., 210 no. 1 (2013), 31-94.

[6] G. Castelnuovo: Sulle transformazioni cremoniane del piano, che ammettono una curva fissa, Rend. Accad. Lincei (1892), Memorie scelte, Zanichelli, Bologna, 1937.

[7] G. Castelnuovo: Le trasformazioni generatrici del gruppo cremoniano nel piano, Atti della R. Accad. delle Scienze di Torino, 36 (1901), 861-874. 
[8] J. Diller, D. Jackson, A. Sommese: Invariant curves for birational surface maps, Trans. Amer. Math. Soc., 359(6) (2007), 2793-2991.

[9] M. H. Gizatullin, The decomposition, inertia and ramification groups in birational geometry, Algebraic Geometry and its Applications, Aspects Math., E25 (1994), 39-45.

[10] I. Hedén, S. Zimmermann, The decomposition group of a line in the plane, (to appear in Proc. Amer. Math. Soc.). Preprint version: arXiv:1601.02725.

[11] A. Lonjou Non simplicité du groupe de Cremona sur tout corps, Ann. Inst. Fourier (Grenoble), 66, no. 5 (2016), 2021-2046.

[12] P. SAmuel Lectures on old and new results on algebraic curves, (notes by S. Amentharsmon) Tata Institute, Colaba, Bombay 5, India (1966).

[13] T. UEHARA, Rational surface automorphisms preserving cuspidal anticanonical curves, Math. Ann., 365, no. 1-2 (2016), 635-659.

Tom Ducat, Research Institute for Mathematical Sciences, Kyoto University, Kyoto 606-8502 JAPAN

E-mail address: taducat@kurims.kyoto-u.ac.jp

Isac Hedén, Research Institute for Mathematical Sciences, Kyoto University, Kyoto 606-8502 JAPAN

E-mail address: Isac.Heden@kurims.kyoto-u.ac.jp

Susanna Zimmermann, Université Toulouse Paul Sabatier, Institut de Mathématiques, 118 route de Narbonne, 31062 Toulouse Cedex 9

E-mail address: susanna.zimmermann@math.univ-toulouse.fr 